\title{
A self-reported Frailty Index predicts long-term mortality in hospitalized patients with cirrhosis
}

\author{
You Deng ${ }^{1, \#}$, Lin Lin ${ }^{3 \#}$, Lijun Hou ${ }^{1,2}$, Xiaofei Fan ${ }^{1,2}$, Tianming Zhao ${ }^{1,2}$, Lihong Mao ${ }^{1,2}$, Xiaoyu Wang ${ }^{1,2}$, \\ Bangmao Wang ${ }^{1,2}$, Yingli Ma ${ }^{1,2}$, Chao Sun ${ }^{1,2,3}$ \\ ${ }^{1}$ Department of Gastroenterology and Hepatology, ${ }^{2}$ Tianjin Institute of Digestive Disease, Tianjin Medical University General Hospital, Tianjin, \\ China; ${ }^{3}$ Department of Gastroenterology, Tianjin Medical University General Hospital Airport Hospital, Tianjin, China \\ Contributions: (I) Conception and design: Y Deng, L Lin, L Hou, X Fan, L Mao, B Wang, Y Ma, C Sun; (II) Administrative support: Y Deng, L \\ Lin, C Sun; (III) Provision of study materials or patients: Y Deng, L Lin, C Sun; (IV) Collection and assembly of data: Y Deng, L Lin, L Hou, T \\ Zhao, L Mao, X Wang, C Sun; (V) Data analysis and interpretation: Y Deng, L Lin, L Hou, Y Ma, C Sun; (VI) Manuscript writing: All authors; \\ (VII) Final approval of manuscript: All authors. \\ \#These authors contributed equally to this work. \\ Correspondence to: Bangmao Wang, MD, PhD; Yingli Ma; Chao Sun, MD, PhD. Department of Gastroenterology and Hepatology, Tianjin Medical University \\ General Hospital, Anshan Road 154, Heping District, Tianjin, China. Email: sch0118@126.com; mylin1970@163.com; chaosun@tmu.edu.cn.
}

Background: Frailty is a syndrome that diminishes the potential for functional recovery in liver cirrhosis (LC). However, its utility is limited due to sole reliance on physical performance, especially in hospitalized patients. We investigate the predictive value of a modified self-reported Frailty Index in cirrhotics, and identify which health deficits play more important roles.

Methods: Consecutive LC patients were assessed by our frailty scale. Outcomes of interest were mortality for 90-day, 1-year and 2-year. Independent predictors were identified by multivariate Cox regression. Receiver operating characteristic curve (ROC) was performed to evaluate discriminative ability. We used a combination of stepwise selection, best subset selection, and Akaike information criteria (AIC) to identify pivotal frailty components.

Results: The study cohort consisted of 158 patients, in which 37 expired during follow-up. Compared with non-frail groups, the frail group had higher 1- and 2-year mortality. The area under ROC of the ChildTurcotte-Pugh classification (CTP) and Frailty Index were 0.66 and 0.68 , while 0.72 for CTP + Frailty Index $(\mathrm{P}=0.034)$, respectively. The optimal predictors comprised instrumental activities of daily living (IADL) limitation, falls and loss of weight $(\mathrm{AIC}=170, \mathrm{C}$-statistic $=0.67)$.

Conclusions: It is plausible for incorporating Frailty Index to improve prognostication in cirrhotics. IADL limitation, falls and loss of weight play more crucial roles on mortality determination.

Keywords: Frailty Index; cirrhosis; mortality; best subset selection

Submitted Jan 20, 2020. Accepted for publication Aug 03, 2020.

doi: $10.21037 /$ atm-20-943

View this article at: http://dx.doi.org/10.21037/atm-20-943

\section{Introduction}

Cirrhosis is associated with remarkable increase of health care costs and the loss of life quality (1), thus efforts to investigate determinants of the rising burden are indispensible. The model for end-stage liver disease (MELD) and Child-Turcotte-Pugh classification (CTP) can facilitate prognostication, however, they fail to capture overall issues contributing to a patient' s clinical circumstance just based on laboratory tests. Frailty is a multidimensional dysfunction state of increased vulnerability to stressors and decline of physiological reserves. And it has been ascertained to predict mortality in not only pretransplant patients but also cirrhotic outpatients.

Frailty used to be defined by either the Fried Frailty 
Criteria (FFC) or Short Physical Performance Battery (SPPB). However, both of them require excessive time to perform, which is not feasible in daily clinical setting (2). Moreover, it is not easy to conduct physical tests in critical cirrhotics with concurrent acute insults. FFC covers five criteria to determine the level of frailty: weight loss, exhaustion, low physical activity, slowness, and weakness. The first three criteria are measured with self-reported questions, while slowness and weakness are assessed by performance-based measures of walk time and handgrip strength. A recent review showed a dramatic variation in how these criteria are estimated among studies (3). These differences lead to variations in frailty prevalence estimates and predictive ability. Therefore, many researchers select questionnaires or a single question instead of performancebased measures.

Carolina Frailty Index (CFI), a 36-item self-reported scale, has demonstrated excellent value to assess frailty in elderly patients with malignancies $(4,5)$. Of note, it is similar with respect to the well-described state of inflammation activation, immune dysfunction, sarcopenia and cachexia in both cancer and cirrhosis. We hypothesized that a modified CFI (thereafter referred to as Frailty Index) could identify frailty in liver cirrhosis (LC). Overall frailty can integrate a variety of health deficits into one single index, which enhance its credibility (6), yet some health deficits among Frailty Index play more important roles in mortality determination of different disease context. Collectively, we intend to assess the prognostic value of Frailty Index in cirrhotics and identify which frailty component impacted most strongly. We present the following article in accordance with the TRIPOD reporting checklist (available at http://dx.doi.org/10.21037/atm-20-943).

\section{Methods}

\section{Study design}

A cohort of consecutive cirrhotic patients was recruited in Tianjin Medical University General Hospital from 2016 to 2017. We identified patients with cirrhosis by collecting medical history, laboratory tests, imaging data, endoscopy results and/or liver biopsy. The exclusion criteria are as follows: (I) unconscious statement or with poor compliance; (II) primary liver cancer or extrahepatic malignancies; (III) more than 26 empty items in the questionnaires; (IV) liver transplantation; (V) refusal to follow-up. At enrollment, all patients underwent the tests of a self-reported Frailty
Index (Figure S1). All assessments were performed by one of our two well trained researchers, who managed these study procedures in the same order and same manner for each participant. Clinical examinations were collected from electronic medical records. All patients were followed prospectively until their terminal event happened. The outcomes of interest were 90 days, 1 year and 2 years mortality. This study was performed in accordance with the ethical standards of the Declaration of Helsinki (as revised in 2013) and was approved by Ethics Committee of Tianjin Medical University General Hospital. Written inform consent was obtained from all participants.

CFI is a self-reported questionnaire consisting of 36-item regarding various aspects: instrumental activities of daily living (IADL), physical function, unintentional weight loss, exhaustion, depression and social activities, etc. (7). We considered the specificity of cirrhosis, modified the CFI and obtained a new Frailty Index. A questionnaire which is fulfilled with 10 items or more will be regarded as valid. The Frailty Index is defined as the score that patients get from the total points of the questionnaire. For instance, a patient who gets 10 points after finishing the all 36 items has a Frailty Index as 0.28 (10/36); another patient who gets 5 points after finishing 15 items of questionnaire has a Frailty Index as $0.33(5 / 15)$.

\section{Statistical approach}

The categorization of Frailty Index was based on quartile. Data were demonstrated as median (interquartile range, IQR) or proportion. Continuous data were compared using an independent Student's $t$-test or the Mann-Whitney U test for groups without normal distribution. Categorical variables were compared by chi-square test or Fisher's exact test as appropriate. Associations between the covariates and mortality were evaluated by using univariate and multivariate Cox regression model. The proportional hazards assumption was assessed by the Kaplan-Meier survival plots. The receiver operating characteristic curve (ROC) was used to evaluate discriminative ability of Frailty Index.

We used stepwise selection, best subset selection, and Akaike information criteria (AIC) reduction in combination to identify frailty components (8). First, stepwise selection identified the full stepwise sequence of potential models from the null model to the full model containing all explanatory variables, for a total of 9 candidate models. We calculated AIC for the 9 models. To assess a broad spectrum 
of models that were predictive in the clinical setting, the best subset selection which ranked models by using the branch-and-bound algorithm was applied. We assessed models with 2 covariates to 6 covariates. Furthermore, we evaluated the 8 best combinations of 2-variable models, 3 -variable models, 4-variable models, 5 -variable models, and 6-variable models. We selected the best models by using AIC and C-statistic simultaneously. Analyses were performed using the R 3.3.2 software package.

\section{Results}

The study cohort consisted of 158 patients. The median age was 64 years (IQR: 57 to 70 years). The etiology of LC was attributed to HBV/HCV infection in 40 (25.3\%), alcohol hepatitis in 24 (15.2\%), autoimmune liver disease in 20 $(12.7 \%)$ and cryptogenic in 74 (46.8\%). Sixty-three subjects were classified as CTP-A, 69 as CTP-B, and 26 as CTP-C, respectively. The mean MELD score at admission was 11 points. Baseline characteristics stratified by vital status are shown in Table 1. Non-survivors had higher CTP (9 vs. 7; $\mathrm{P}<0.001$ ), MELD Score (13 vs. 11; $\mathrm{P}=0.001)$, TBIL (44.7 vs. $28.3 \mu \mathrm{mol} / \mathrm{L} ; \mathrm{P}=0.018)$, creatinine (91 vs. $64 \mu \mathrm{mol} / \mathrm{L}$; $\mathrm{P}<0.001)$, Frailty Index (0.28 vs. $0.17 ; \mathrm{P}<0.001)$, lower serum albumin (28 vs. $32 \mathrm{~g} / \mathrm{L} ; \mathrm{P}=0.003)$ and lymphocytes to monocytes ratio (1.8 vs. 2.6; $\mathrm{P}=0.031$ ).

Basing on quartile, we defined the Frailty Index that less than 0.07 as robust, $0.07-0.38$ as pre-frail (we classified robust and pre-frail as non-frail phenotype for analytic convenience) and more than 0.38 as frail. Non-survivors had higher levels of Frailty Index ( $\mathrm{P}=0.011)$, as shown in Table 2. Non-survivors also had an aggravated IADL limitation (0.38 vs. $0.19 ; \mathrm{P}=0.003)$, impaired physical function $(0.45$ vs. 0.28; $\mathrm{P}=0.027)$, a higher prevalence of falls $(30 \%$ vs. $9 \%$; $\mathrm{P}=0.005)$ and hearing impairment (35\% vs. $18 \% ; \mathrm{P}=0.041)$. There was no significant difference in exhaustion $(59 \%$ vs. $40 \% ; \mathrm{P}=0.058)$, depression $(54 \%$ vs. $41 \% ; \mathrm{P}=0.190)$, medications ( $8 \%$ vs. $3 \% ; \mathrm{P}=0.356)$, comorbidities $(68 \%$ vs. $57 \% ; \mathrm{P}=0.339)$, visual impairment ( $35 \%$ vs. $26 \% ; \mathrm{P}=0.296)$, loss of weight $(24 \% v s .13 \% ; \mathrm{P}=0.124)$ and social activity (65\% vs. $47 \%$; $\mathrm{P}=0.063$ ).

There was no significant difference in 90-day mortality ( $10.3 \%$ vs. $3.3 \%, \mathrm{P}=0.104$ ) between the frail group and the non-frail group. In contrast, the frail group had a significant increase in 1-year $(30.8 \%$ vs. $11.8 \%, \mathrm{P}=0.011)$ and 2-year (41\% vs. $17.6 \%, \mathrm{P}=0.0044)$ mortality (Figure 1 ). The Kaplan-Meier survival curves (Figure 2) showed significant difference between the frail and non-frail group $(\mathrm{P}=0.001)$.
In univariate analysis, Frailty Index was a risk factor for 2-year mortality in cirrhosis. In both multivariate analysis models, Frailty Index (\#model 1 HR: 4.70; 95\% CI: 1.40 to 15.74 ; \#model 2 HR: 5.95 ; $95 \%$ CI: 1.80 to 19.73 ) demonstrated significant association with 2-year mortality (Table 3). The ability of CTP and Frailty Index to correctly rank patients according to their 2-year mortality risk were 0.66 and 0.68 , respectively, while 0.72 for CTP + Frailty Index, and 0.67 for MELD, 0.72 for MELD + Frailty Index (Figure 3).

Stepwise selection identified the optimal three variables including IADL, falls and loss of weight. The best subset selection ranked models by using the branch-and-bound algorithm, thus the best model comprised six variables including IADL, physical function, falls, hearing, loss of weight and social activity which encompassed the highest adjusted R-square (Figure 4A). Furthermore, the number of variables in best model was decided by Mallow $\mathrm{Cp}$ statistic. It suggested that I-F-L model, I-F-H-L model, I-P-F-H-L model and I-P-F-H-L-S model maybe become the "best" models (Figure 4B). The AIC and C-statistic of eight candidate models of 2 -variable models, 3 -variable models, 4-variable models, 5-variable models, and 6-variable models were calculated and shown in Table 4. In accordance with the low AIC and high C-statistic, we believe that the IADL, falls and loss of weight play more important roles in mortality determination in cirrhotics.

\section{Discussion}

Frailty challenges healthcare professionals and has pervasive impact on health as well as the outcomes of health care. It has been proposed that frailty should always be taken into account when treating the older patients. FFC and SPPB have been developed to measure frailty. However, both of them require physical frailty components. These physical tests have reduced applicability in more severe patients with complications, as well as in critically ill inpatient setting (9). On the other hand, when screening large populations, performance-based measures can be difficult to conduct because they are time consuming and costly, and often require well-trained practitioners. In a word, questionnaires instead of performance-based measures seem suitable, and CFI has shown excellent value in identifying frailty in elderly patients with malignancies. Furthermore, sarcopenia and systemic inflammation have previously been shown to correlate with prognosis in both cancer and cirrhosis $(10,11)$. Therefore, we evaluated the predictive value of 
Table 1 Baseline characteristics of recruited patients with cirrhosis

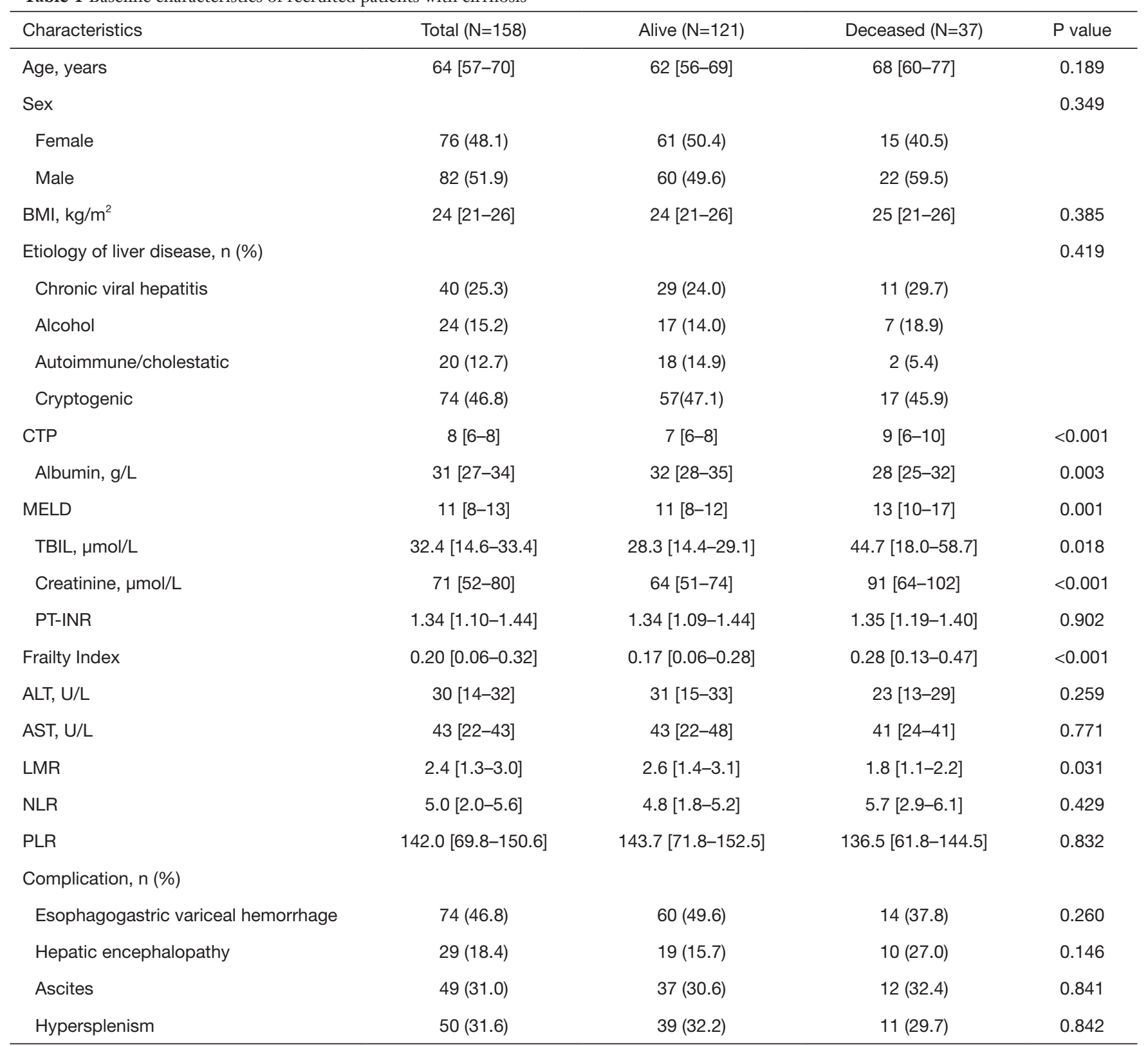

Values are median [IQR] or n (\%). BMI, body mass index; CTP, Child-Turcotte-Pugh classification; MELD, model for end-stage liver disease; TBIL, total bilirubin; PT-INR, international normalized ratio for prothrombin time; ALT, alanine aminotransferase; AST, aspartate aminotransferase; LMR, lymphocyte monocyte ratio; NLR, neutrophil lymphocyte ratio; PLR, platelet lymphocyte ratio.

Frailty Index, which is developed from the CFI, in our well-established cohort of patients with cirrhosis. Our results suggested that the Frailty Index could predict poor outcomes effectively in cirrhotics. Notably, Frailty Index was an independent predictor of 1- and 2-year mortality in hospitalized patients with cirrhosis and it could complement the CTP or MELD in clinical decision-making. Moreover,
IADL limitation, falls and loss of weight play more important roles in mortality determination for patients with cirrhosis.

To the best of our knowledge, this is the first study to assess the predictive ability of self-reported frailty with respect to long-term mortality in hospitalized LC patients. Several frailty scoring tools have been proposed to predict 
Table 2 Baseline self-reported Frailty Index-related characteristics

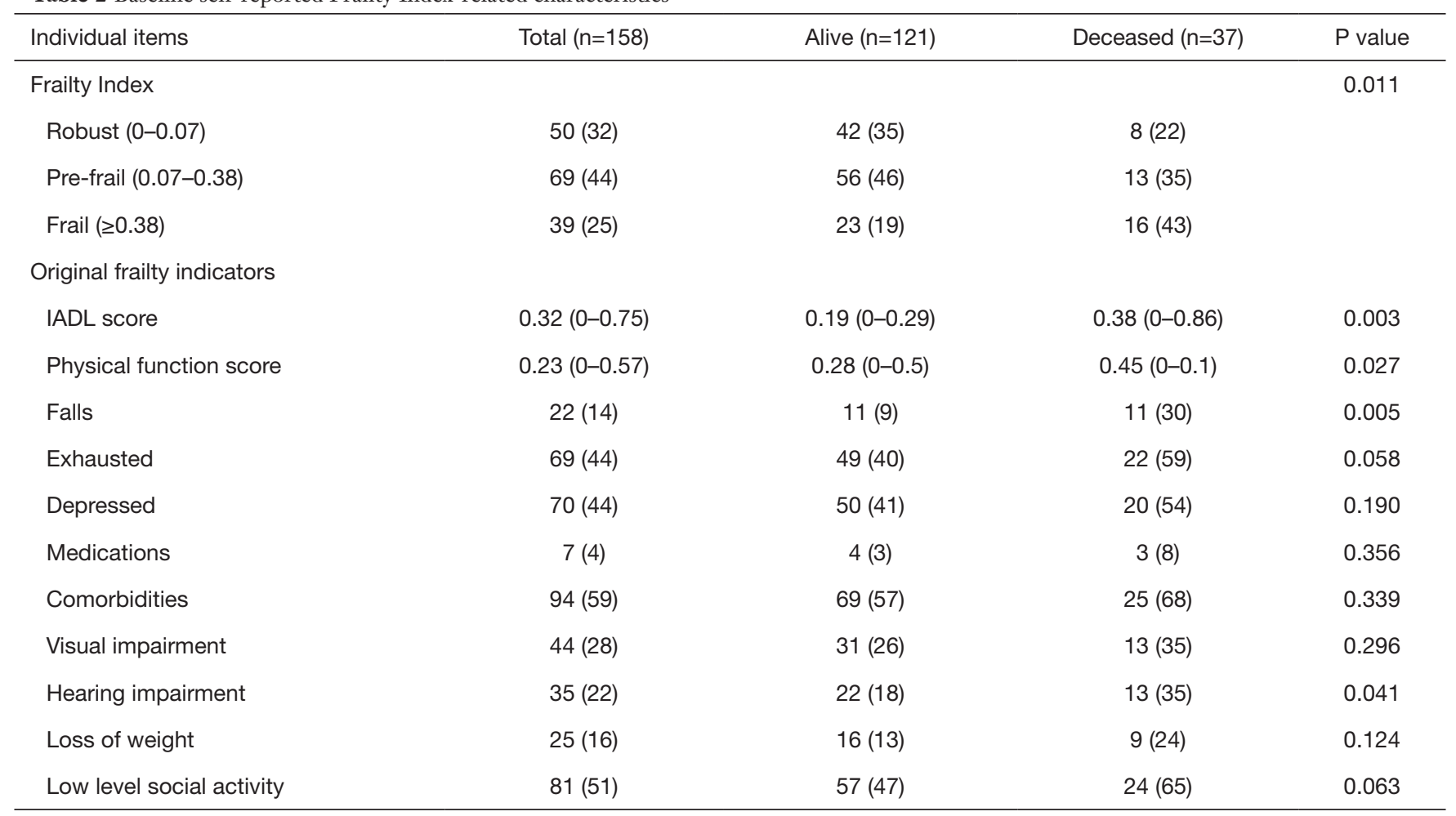

Values are median (IQR) or n (\%). For the Frailty Index, higher scores indicate greater frailty. IADL, Instrumental activities of daily living.

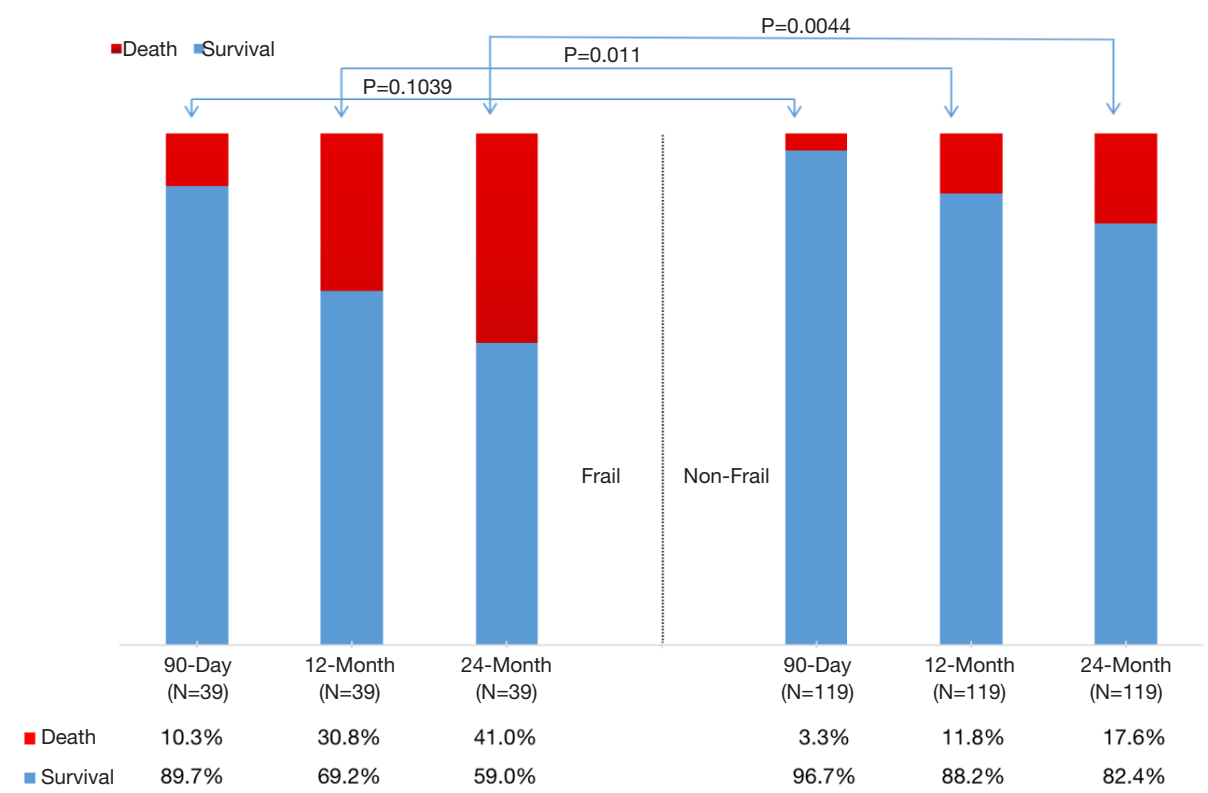

Figure 1 The overall mortality of frail and non-frail group at 90 days, 12 months and 24 months. The bar subdivisions represent the proportion of patients expired from any cause (red) and survival (blue). 

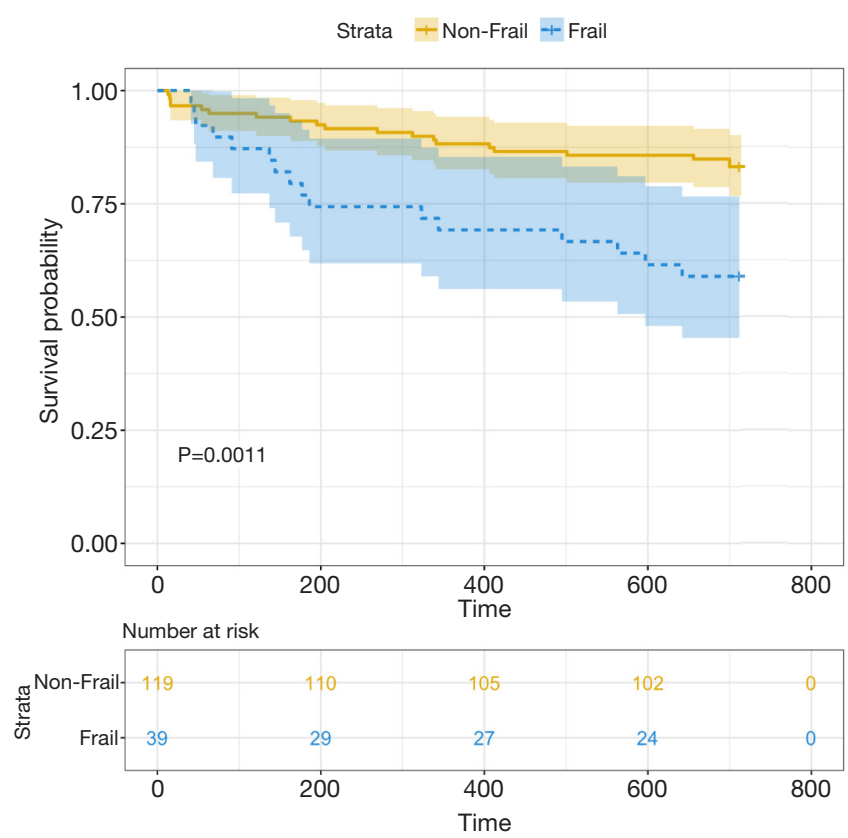

Figure 2 Predicted probabilities of survival for patients with cirrhosis, classified by Frailty Index. The categorization of Frailty Index was based on quartile (we defined the Frailty Index that less than 0.07 as robust, $0.07-0.38$ as pre-frail and more than 0.38 as frail, we classified robust and pre-frail as non-frail phenotype for analytic convenience).

adverse clinical outcomes in outpatients with cirrhosis. Frailty has recently been validated to predict 90-day and 1 -year mortality in the pre-transplants population and cirrhotic outpatients $(8,12)$. Tapper et al. suggested that standard assessments of frailty are significant predictors of 90-day mortality in hospitalized patients with cirrhosis (13). Our study also demonstrated that the 90-day mortality of frail group increased compared with non-frail group but without significant difference $(\mathrm{P}=0.104)$. Frailty represents a chronic low-grade inflammatory state with immune dysfunction, which is similar to that in hospitalized cirrhotic patients. We suppose it is more reasonable to evaluate the long-term mortality, and our results showed Frailty Index is independently associated with 1-year and 2-year mortality.

Adding the Frailty Index to the MELD and CTP may be a favorable manner to make clinical decision. MELD and CTP score have been recognized as predictors of survival in patients with cirrhosis. However, there are approximately $15-20 \%$ of patients whose survival cannot be accurately predicted (14), since traditional scoring systems fail to capture the features of muscle wasting, malnutrition, and
Table 3 Univariable and multivariable analysis to predict 2-yr mortality in cirrhosis

\begin{tabular}{lcc}
\hline Variables & HR $(95 \% \mathrm{Cl})$ & $\mathrm{P}$ value \\
\hline Univariate analysis & $1.58(0.79-3.18)$ & 0.186 \\
Age & $0.65(0.33-1.28)$ & 0.206 \\
Sex & $1.31(1.15-1.50)$ & $<0.001$ \\
CTP & $1.11(1.04-1.18)$ & 0.003 \\
MELD & $8.99(2.80-28.88)$ & 0.001 \\
Frailty Index & $0.69(0.50-0.95)$ & 0.008 \\
LMR & & \\
Multivariate analysis & & \\
\# model 1 & & \\
Age & & 0.003 \\
Sex & & \\
CTP & $1.26(1.08-1.46)$ & \\
Frailty Index & $4.70(1.40-15.74)$ & \\
LMR & & 0.012 \\
\# model 2 & & \\
Age & & \\
Sex & & \\
MELD & & \\
Frailty Index & & \\
LMR & & \\
\hline
\end{tabular}

CTP, Child-Turcotte-Pugh classification; MELD, model for endstage liver disease; LMR, lymphocyte monocyte ratio.

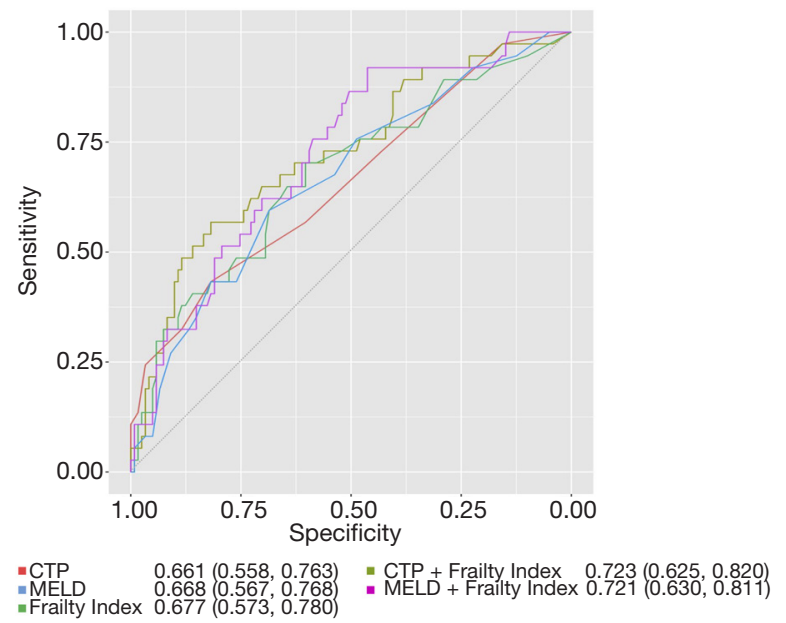

Figure 3 The Frailty Index was added to CTP and MELD. Receiver operating characteristic (ROC) curves were plotted. The inset figure legends display the C-statistic and $95 \%$ confidence interval for each one. CTP, Child-Turcotte-Pugh classification; MELD, model for end-stage liver disease. 


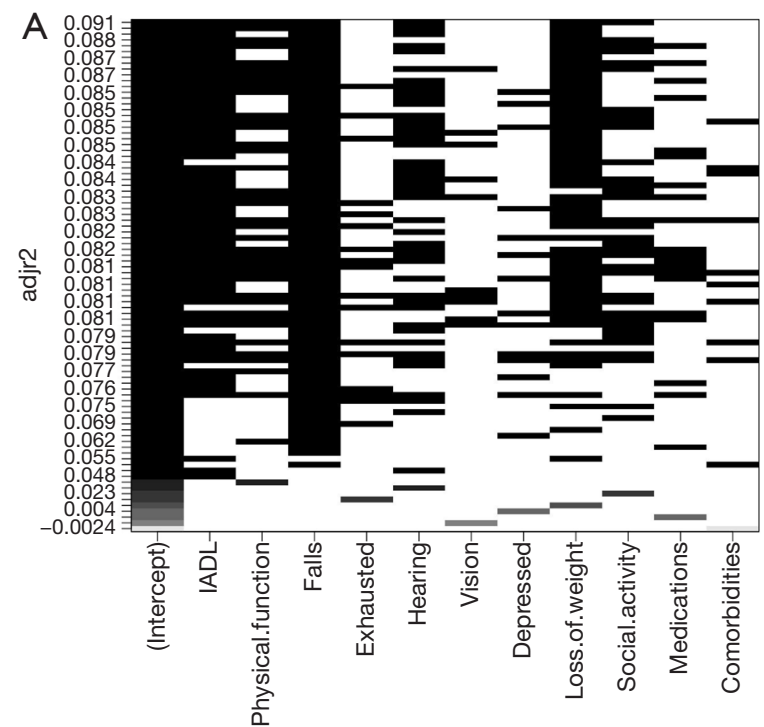

B Cp Plot for All Subsets Regression

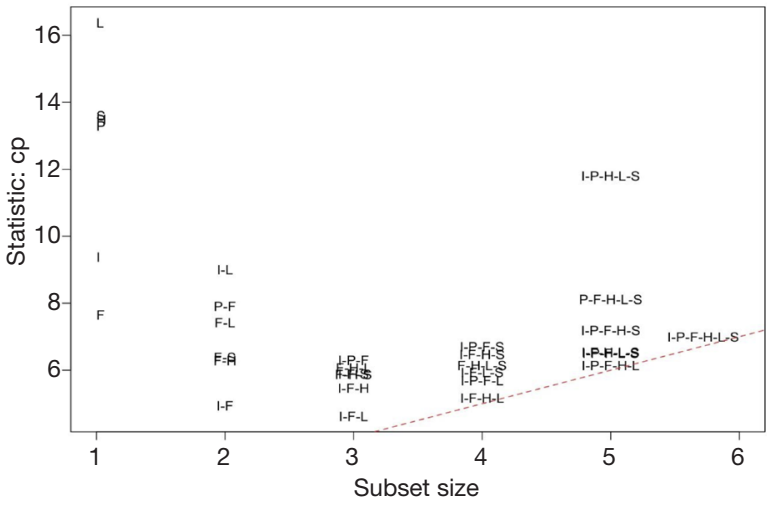

Figure 4 Variable selection results from best subset analyses exploring the importance of frailty components. (A) The number of predictors was decided by adjusted R2. Each column corresponds to a frailty variables. Each row corresponds to a model (i.e., combination of variables), which were ranked using the branch-and-bound algorithm in order of global score chi-square statistic. By adjusted R2, the best model includes IADL, physical function, falls, hearing, loss of weight and social activity (variables that have black boxes at the highest Y-axis value). (B) The number of predictors was decided by Mallow Cp statistic. The stopping rule is to start with the smallest model and gradually increase number of variables, and stop when Mallow Cp is approximately (number of regressors +1 , broken line) for the first time. In this case, the model with 3 regressors (I-F-L) is the first one to achieve such a condition. IDAL, instrumental activities of daily living.

Table 4 Candidate models incorporating various

\begin{tabular}{|c|c|c|c|c|c|c|c|c|}
\hline Variables & \multicolumn{8}{|c|}{ Number of variables included } \\
\hline Mode I\# & $\# 1$ & \#2 & \#3 & $\# 4$ & $\# 5$ & \#6 & \#7 & \#8 \\
\hline IADL & $\sqrt{ }$ & $\sqrt{ }$ & $\sqrt{ }$ & $\sqrt{ }$ & $\sqrt{ }$ & $\sqrt{ }$ & $\sqrt{ }$ & $\sqrt{ }$ \\
\hline Falls & $\sqrt{ }$ & $\sqrt{ }$ & $\sqrt{ }$ & $\sqrt{ }$ & $\sqrt{ }$ & $\sqrt{ }$ & $\sqrt{ }$ & $\sqrt{ }$ \\
\hline Social activities & & & $\sqrt{ }$ & & & $\sqrt{ }$ & & $\sqrt{ }$ \\
\hline Loss of weight & & $\sqrt{ }$ & & $\sqrt{ }$ & $\sqrt{ }$ & $\sqrt{ }$ & $\sqrt{ }$ & $\sqrt{ }$ \\
\hline Hearing & & & & $\sqrt{ }$ & & & $\sqrt{ }$ & $\sqrt{ }$ \\
\hline AIC & 170 & 170 & 171 & 170 & 170 & 171 & 171 & 172 \\
\hline
\end{tabular}

As a point of reference, in our cohort, the AIC and C-statistic for CTP was 168 and 0.66, for MELD was 172 and 0.67, for Frailty Index was 170 and 0.68 . Higher AIC indicates relative lower model quality. IADL, instrumental activities of daily living; AIC, Akaike information criteria. 
functional decline (15). Furthermore, the MELD and CTP scores focus on physiological parameters which are built on creatinine, bilirubin and other indicators, while no effective ways are always anticipated to reverse MELD or CTP score. On contrast, frailty may be a modifiable risk factor responding to early and planned nutritional support as well as physical exercise.

Among different population, the effects of frailty components on mortality were different. Yang et al. demonstrated that IADL and ADL limitations play greater roles in mortality in older people (16). It has also been reported that a greater contribution of cognitive impairment to mortality is observed in women than in men (17). Lai et al. examined that 3 performance-based metrics (grip strength, chair stands and balance) play important roles in pre-transplant outpatients (8). In the current study, we implicated that IADL limitation, falls and loss of weight impact most strongly on all-cause mortality. By uncovering these, interventions such as exercise, falls prevention and nutrition supplement might be directed towards narrowing the gap between patients with a higher versus a lower longterm mortality (18).

Most falls in cirrhosis are attributable to a coalescence of discrete subclinical impairments (19). There are complex potential mechanisms driving fall risk in cirrhosis as follows: cognitive dysfunction, hepatic encephalopathy, alcoholic intoxication and poor environmental awareness stemming from neuropathy alter proprioception (20). Furthermore, ascites alter the center of gravity and give rise to nutritional deficiencies, which may worsen the sense of perception and balance (21). Meanwhile, falls considered to be a 'geriatric syndrome' are rooted in the frailty/sarcopenia process (22). IADL limitation, which describes the ability to independently adapt to the environment, is an enhancement to physical function. Tolea et al. suggested that the ability to perform IADL is one of the major predictors of participation in daily life, and its decline is one of the early precursors of basic functional challenges in adults (23). A systematic review addressed that frail people are more likely to develop or present with IADL disabilities (24). Teo et al. found that the prevalence of IADL disability increased 5- to 11-fold in frail individuals (25). Loss of weight was reported to be associated with increased morbidity and mortality (26). There is ample evidence showing that weight loss and muscle loss closely correlate with frailty, loss of independence, poor prognosis and increased mortality (27).

Our study still has some limitations. First, this study took place on a dedicated liver disease unit in a single center, therefore population bias might exist. Our Frailty Index, which is developed for patients with end-stage liver disease, allows us to converge on a relatively parsimonious number of individual tests of physical frailty. However, multicenter studies should be conducted to better understand the impact of frailty in cirrhotics. Second, our Frailty Index was a self-reported scale, including only subjective indicators. As a matter of fact, our team is measuring 5-meter gait speed as an indicator of physical frailty for prognostication in cirrhotics, and preliminary results will be present in future publication.

In conclusion, simple assessment of self-reported frailty at admission could predict long-term mortality in patients with cirrhosis.

\section{Acknowledgments}

Funding: This work was partly supported by Tianjin Research Innovation Project for Postgraduate Students (2019YJSS186) to TZ.

\section{Footnote}

Reporting Checklist: The authors have completed the TRIPOD reporting checklist. Available at http://dx.doi. org/10.21037/atm-20-943

Data Sharing Statement: Available at http://dx.doi. org/10.21037/atm-20-943

Peer Review File: Available at http://dx.doi.org/10.21037/ atm-20-943

Conflicts of Interest: All authors have completed the ICMJE uniform disclosure form (available at http://dx.doi. org/10.21037/atm-20-943). The authors have no conflicts of interest to declare.

Ethical Statement: The authors are accountable for all aspects of the work in ensuring that questions related to the accuracy or integrity of any part of the work are appropriately investigated and resolved. This study was performed in accordance with the ethical standards of the Declaration of Helsinki (as revised in 2013) and was approved by Ethics Committee of Tianjin Medical University General Hospital (2015-024). Written inform consent was obtained from all participants. 
Open Access Statement: This is an Open Access article distributed in accordance with the Creative Commons Attribution-NonCommercial-NoDerivs 4.0 International License (CC BY-NC-ND 4.0), which permits the noncommercial replication and distribution of the article with the strict proviso that no changes or edits are made and the original work is properly cited (including links to both the formal publication through the relevant DOI and the license). See: https://creativecommons.org/licenses/by-nc-nd/4.0/.

\section{References}

1. Stepanova M, De Avila L, Afendy M, et al. Direct and indirect economic burden of chronic liver disease in the United States. Clin Gastroenterol Hepatol 2017;15:759-66.e5.

2. Sze $\mathrm{S}$, Pellicori $\mathrm{P}$, Zhang J, et al. Identification of frailty in chronic heart failure. JACC Heart Fail 2019;7:291-302.

3. Theou O, Cannl L, Blodgett J, et al. Modifications to the frailty phenotype criteria: Systematic review of the current literature and investigation of 262 frailty phenotypes in the Survey of Health, Ageing, and Retirement in Europe. Ageing Res Rev 2015;21:78-94.

4. Guerard EJ, Deal AM, Chang Y, et al. Frailty Index Developed From a Cancer-Specific Geriatric Assessment and the Association With Mortality Among Older Adults With Cancer. J Natl Compr Canc Netw 2017;15:894-902.

5. Williams GR, Deal AM, Muss HB, et al. Frailty and skeletal muscle in older adults with cancer. J Geriatr Oncol 2018;9:68-73.

6. Theou O, Rockwood MR, Mitnitski A, et al. Disability and co-morbidity in relation to frailty: how much do they overlap? Arch Gerontol Geriatr 2012;55:e1-8.

7. Nishijima TF, Deal AM, Williams GR, et al. Frailty and inflammatory markers in older adults with cancer. Aging (Albany NY) 2017;9:650-64.

8. Lai JC, Covinsky KE, Dodge JL, et al. Development of a novel frailty index to predict mortality in patients with end-stage liver disease. Hepatology 2017;66:564-74.

9. Laube R, Wang H, Park L, et al. Frailty in advanced liver disease. Liver Int 2018;38:2117-28.

10. Montano-Loza AJ, Meza-Junco J, Prado CM, et al. Muscle wasting is associated with mortality in patients with cirrhosis. Clin Gastroenterol Hepatol 2012;10:16673, $73 . e 1$.

11. Feliciano EMC, Kroenke CH, Meyerhardt JA, et al. Association of Systemic Inflammation and Sarcopenia With Survival in Nonmetastatic Colorectal Cancer: Results
From the C SCANS Study. JAMA Oncol 2017;3:e172319.

12. Lai JC, Covinsky KE, McCulloch CE, et al. The Liver Frailty Index Improves Mortality Prediction of the Subjective Clinician Assessment in Patients With Cirrhosis. Am J Gastroenterol 2018;113:235-42.

13. Tapper EB, Finkelstein D, Mittleman MA, et al. Standard assessments of frailty are validated predictors of mortality in hospitalized patients with cirrhosis. Hepatology 2015;62:584-90.

14. Kamath PS, Kim WR, Advanced Liver Disease Study Group. The model for end-stage liver disease (MELD). Hepatology 2007;45:797-805.

15. van Vugt JLA, Alferink LJM, Buettner S, et al. A model including sarcopenia surpasses the MELD score in predicting waiting list mortality in cirrhotic liver transplant candidates: A competing risk analysis in a national cohort. J Hepatol 2018;68:707-14.

16. Yang F, Gu D. Predictability of frailty index and its components on mortality in older adults in China. BMC Geriatr 2016;16:145.

17. Shi J, Yang $Z$, Song $X$, et al. Sex differences in the limit to deficit accumulation in late middle-aged and older Chinese people: results from the Beijing Longitudinal Study of Aging. J Gerontol A Biol Sci Med Sci 2014;69:702-9.

18. Ney M, Vandermeer B, van Zanten SJ, et al. Meta-analysis: oral or enteral nutritional supplementation in cirrhosis. Aliment Pharmacol Ther 2013;37:672-9.

19. Murphy SL, Tapper EB, Blackwood J, et al. Why Do Individuals with Cirrhosis Fall? A Mechanistic Model for Fall Assessment, Treatment, and Research. Dig Dis Sci 2019;64:316-23.

20. Román E, Cordoba J, Torrens M, et al. Minimal hepatic encephalopathy is associated with falls. Am J Gastroenterol 2011;106:476-82.

21. Tapper EB, Risech-Neyman Y, Sengupta N. Psychoactive Medications Increase the Risk of Falls and Fall-related Injuries in Hospitalized Patients With Cirrhosis. Clin Gastroenterol Hepatol 2015;13:1670-5.

22. Inouye SK, Studenski S, Tinetti ME, et al. Geriatric syndromes: clinical, research, and policy implications of a core geriatric concept. J Am Geriatr Soc 2007;55:780-91.

23. Tolea MI, Morris JC, Galvin JE. Longitudinal associations between physical and cognitive performance among community-dwelling older adults. PLoS One 2015;10:e122878.

24. Kojima G. Frailty as a predictor of disabilities among community-dwelling older people: a systematic review and meta-analysis. Disabil Rehabil 2017;39:1897-908. 
25. Teo N, Gao Q, Nyunt MSZ, et al. Social Frailty and Functional Disability: Findings From the Singapore Longitudinal Ageing Studies. J Am Med Dir Assoc 2017;18:637.e13-e19.

26. Alibhai SM, Greenwood C, Payette H. An approach to

Cite this article as: Deng $\mathrm{Y}$, Lin L, Hou L, Fan X, Zhao T, Mao L, Wang X, Wang B, Ma Y, Sun C. A self-reported Frailty Index predicts long-term mortality in hospitalized patients with cirrhosis. Ann Transl Med 2020;8(19):1217. doi: 10.21037/atm20-943 the management of unintentional weight loss in elderly people. CMAJ 2005;172:773-80.

27. Ali S, Garcia JM. Sarcopenia, cachexia and aging: diagnosis, mechanisms and therapeutic options - a minireview. Gerontology 2014;60:294-305. 